\title{
Differential Response of Common Bean Genotypes to High Temperature
}

\author{
K.M. Rainey and P.D. Griffiths ${ }^{1}$ \\ Department of Horticultural Sciences, Cornell University NYSAES, Geneva, NY 14456
}

ADDITIONAL INDEX WORDs. breeding, Phaseolus vulgaris, snap bean, temperature stress

\begin{abstract}
Yield components of 24 common bean (Phaseolus vulgaris L.) genotypes were evaluated following exposure during reproductive development to four greenhouse day/night temperature treatments $\left(24^{\circ} \mathrm{C} / 21^{\circ} \mathrm{C}, 27^{\circ} \mathrm{C} / 24^{\circ} \mathrm{C}, 30\right.$ ${ }^{\circ} \mathrm{C} / 27^{\circ} \mathrm{C}$ and $33^{\circ} \mathrm{C} / 30{ }^{\circ} \mathrm{C}$ ). Genotypes included 12 snap beans, two wax beans, six dry beans, and four common bean accessions; 18 genotypes were previously described as heat-tolerant and three were heat-sensitive controls. The highest temperature treatment reduced seed number, pod number, mean seed weight and seeds/pod an average of $83 \%$, $63 \%$, $47 \%$, and $73 \%$, respectively. A heat susceptibility index (S) measuring yield stability under high temperatures indicated that 'Brio', 'Carson', 'G122', 'HB 1880', 'HT 20', 'HT 38', 'Opus', and 'Venture' were heat tolerant. Heat-tolerant genotypes displayed differential responses to high temperature, suggesting different genetic control of heat tolerance mechanisms. Genotypes with moderate heat tolerance, including 'Barrier' and 'Hystyle', showed stable yields in the $30{ }^{\circ} \mathrm{C} / 27^{\circ} \mathrm{C}$ treatment only, indicating this regime is optimal for screening common bean materials of unknown heat tolerance. 'Haibushi', 'Indeterminate Jamaica Red', and 'Tío Canela-75' were previously described as heat tolerant but exhibited a heat-sensitive reaction in this study. Heat-sensitive genotypes 'Haibushi' and 'Labrador' maintained mean seed weight under high temperature. This data will help utilize nonallelic heat tolerance genes in development of bean varieties grown in high temperature environments.
\end{abstract}

Heat sensitivity is a major limiting factor in the production of common beans, causing reduced yields, reduced product quality, and restricted geographic adaptation. Temperate common bean production areas experience brief and problematic seasonal heat waves during flowering, resulting in blossom drop, and in the case of snap bean, a split set. Also, distally located ovules fail to develop, reducing seed number in dry bean and product quality in snap bean due to misshapen pods (Myers and Baggett, 1999). By contrast, high temperatures in the tropics restrict common bean cultivation at lower elevations and in warmer seasons ( $>25$ ${ }^{\circ} \mathrm{C}$ ) (Baiges et al., 1996; Porch and Jahn, 2001; Schoonhoven and Voysest, 1991; Weaver et al., 1985). Improving heat tolerance in common bean (both dry and snap) increases yield stability and extends the geographic range of cultivation. The latter is especially important in the tropics at lower elevations (Porch, 2001).

Heat-tolerant common bean genotypes have been developed or identified from diverse gene pools and origins. Studies have compared responses of heat-tolerant and -sensitive genotypes (Gross and Kigel, 1994; Monterroso and Wien, 1990; Porch and Jahn, 2001), and demonstrate that genetic variability exists for heat tolerance in common bean. Many sources report the dry bean 'G122' (Shonnard and Gepts, 1994) possesses a high level of heat tolerance; however, in attempts to transfer this tolerance, this genotype does not combine well with other common bean genetic backgrounds (Porch, 2001). Heat-tolerant dry bean varieties have recently been released and include 'Indeterminate Jamaica Red' ('IJR') (Baiges et al., 1996) and 'Tío Canela-75' (Rosas et al., 1997). Heat-tolerant snap bean varieties include 'Venture' (Dickson, personal communication), 'Brio' and 'Opus'. 'Haibushi' was developed as a heat-tolerant indeterminate snap bean from

Received for publication 19 June 2003. Accepted for publication 1 June 2004. I thank Mike Dickson, Molly Jahn, George Kotch, Ron Riley, and Rusty Smith for help in obtaining the genotypes used in this work.

1To whom reprint requests should be addressed: phone: 315-787-2222; Fax: 315-787-2216; e-mail: pdg8@cornell.edu. south Asian germplasm (Suzuki et al., 2001). Wien and Munger (1972) identified USDA accessions PI 271997 and PI 271998 as heat tolerant. Many other genotypes are reported heat tolerant based on personal communication or cultivar descriptions.

It has been established that the greatest yield losses occur under conditions of high night temperatures, which negatively affect all stages of reproductive development in common bean. To date, studies of high-temperature effects on yield in common bean have focused on the timing of application of temperature stress in relation to specific developmental processes. Microsporogenesis (occurring 6-12 d prior to anthesis) was found to be the most sensitive developmental stage (Gross and Kigel, 1994). Many studies have associated reduced pollen viability with high temperature response in common bean, and a correlation between production of inviable pollen and reduction of yield components has been established (Halterlein et al., 1980; Suzuki et al. 2001; Weaver et al., 1985). Reduced yields have been associated with morphological abnormalities in common bean pollen and anthers produced under high temperatures (Gross and Kigel, 1994; Porch and Jahn, 2001; Suzuki et al., 2001). Production of parthenocarpic pods in common bean is also attributed to high temperatures during sporogenesis (Gross and Kigel, 1994).

Abscission of reproductive organs can be the primary determinant of pod number under heat stress (Ofir et al., 1993). Studies have shown that high temperatures applied during sporogenesis result in the highest rates abscission (Monterroso and Wien, 1990). Flowers are the most likely reproductive organs to abscise, followed by young pods ( $<2 \mathrm{~cm}$ long) and then buds, indicating that abscission of more mature organs results from a failure of fertilization or seed set (Konsens et al., 1991; Monterroso and Wien, 1990). In contrast to nonstressed plants, common beans under heat stress produce more flower primordia and a relatively higher proportion of these abscise, either as primordia or more mature organs (Konsens et al., 1991).

High temperatures impair other aspects of reproductive development in common bean. Observation of ovule positions in relation to seed set, reciprocal pollinations, and histological 
examinations have indicated that pollen tube growth and fertilization are inhibited by high temperatures (Dickson and Boettger, 1984; Gross and Kigel, 1994; Halterlein et al., 1980; Omrod et al., 1967). Reciprocal pollinations and imposition of heat stress shortly before and after anthesis showed reduced gynoecium function under heat stress in common bean as well (Gross and Kigel, 1994; Monterroso and Wien, 1990).

While previous studies have evaluated effects of high temperature on specific stages of reproductive development, there have been no studies characterizing response to high temperatures of a variety of heat-tolerant common bean materials in large-scale controlled environments. Identifying differential heat-tolerance responses may allow for the combination of nonallelic heattolerance genes in order to improve yields in high-temperature environments. Studies of temperature stress in cowpea [Vigna unguiculata (L.) Walp.] showed that relative plant performance under high-temperature greenhouse conditions was effective in identifying genotypes that displayed heat tolerance under field conditions (Ehlers and Hall, 1996; Ismail and Hall, 1998).

The objectives of this study were to 1) characterize 24 common bean genotypes for yield and its components under four temperature treatments and 2) determine optimal temperature regimes for determining heat tolerance and heat sensitivity of common bean genotypes in controlled environments.

\section{Materials and Methods}

Plant materials. Twenty-four snap and dry bean genotypes were included in the experiment (Table 1). 'HT 20', 'HT 24', and 'HT 38', and PI 182004, PI 307802, and PI 309844 were identified by the authors as heat tolerant in our previous studies. Seeds were sown in four $90-\mathrm{m}^{2}$ greenhouses on 16 Oct. 2002 in $14.6 \times 14.6-\mathrm{cm}$ square pots filled with "Cornell mix" (Boodley and Sheldrake, 1972). Plants were irrigated as needed and fertilized equally in all four greenhouses.

Greenhouse SetTings. Greenhouses were set for a 14-h photoperiod with supplemental lighting provided by $1000-\mathrm{W}$ metal halide bulbs (SunSystem III from Sunlight Supply, Vancouver, Wash.) at a rate of $300 \mu \mathrm{mol} \cdot \mathrm{m}^{-2} \cdot \mathrm{s}^{-1}$ of photosynthetically active radiation at bench level. Greenhouse temperatures were set at 24 ${ }^{\circ} \mathrm{C}$ day $/ 21^{\circ} \mathrm{C}$ night for germination and development of seedlings. Heat treatment was applied $7 \mathrm{~d}$ prior to the earliest anthesis on 5 Nov. 2002. Greenhouse 1 (T1) settings were maintained at $24^{\circ} \mathrm{C}$ day $/ 21^{\circ} \mathrm{C}$ night and greenhouses 2 through $4(\mathrm{~T} 2, \mathrm{~T} 3, \mathrm{~T} 4)$ were reset to $27^{\circ} \mathrm{C} / 24^{\circ} \mathrm{C}, 30^{\circ} \mathrm{C} / 27^{\circ} \mathrm{C}$, and $33^{\circ} \mathrm{C} / 30^{\circ} \mathrm{C}$, respectively. Uniformity of temperatures was measured using a CR21X datalogger with 17 thermocouples spread evenly throughout replications in each treatment at different points in time (Campbell Scientific, Logan, Utah). Heat treatment ceased when plants had begun to senesce on 10 Dec. 2002.

EXPERIMENTALDESIGN. Genotypes were planted in a randomized block design with four replications and five plants per genotype in each replication in all four greenhouses. The same randomization design was used for all four treatments to ensure consistent positional effects for genotypes across the four temperature regimes. Guard rows were used to surround the experiments to maintain uniform light and temperature distribution, microclimate humidity and air movement.

Data Collection. After plants had dried down on 29 Dec. 2002, all pods were harvested from individual plants. Pod and seed counts and seed weight (to $1 \times 10^{-2} \mathrm{~g}$ ) were recorded separately for each plant in the experiment. Aborted ovules, defined as seeds too small to be felt with fingertips through the crushed walls of a dried pod, were not counted. Culls (defined as seeds weighing $<0.10 \mathrm{~g}$ ) were identified, counted, and weighed separately from fully developed seeds. For this analysis, culls were included in the seed number and mean seed weight calculations. Pins were defined as pods not possessing any seeds and were counted separately from pods. Abscission was estimated by visually rating the mean number of reproductive organs per plant for each genotype in each replication and treatment 7 and $14 \mathrm{~d}$ after flowering commenced.

Data Analysis. Yield component data was analyzed using the general linear model analysis, where type III sums of squares comparisons were used to obtain $\mathrm{F}$ values for experimental variables, and means comparisons of yield components were calculated using Duncan's multiple range test at $P \leq 0.05$ (SAS Inst., 1997).

Heat susceptibility index $(\mathrm{S})$ was calculated using the formula of Fischer and Maurer (1978): $S=\left(1-Y / Y_{p}\right) D$.

where $\mathrm{Y}=$ mean yield or yield component of a genotype under heat stress $(\mathrm{T} 3+\mathrm{T} 4)$

$Y_{p}=\quad$ mean yield or yield component of a genotype without heat stress $(\mathrm{T} 1+\mathrm{T} 2)$

$\mathrm{D}=\quad$ stress intensity $=1-\mathrm{X}_{\mathrm{p}} / \mathrm{X}$

$\mathrm{X}=\quad$ mean $\mathrm{Y}$ of all genotypes

$\mathrm{X}_{\mathrm{p}}=$ mean $\mathrm{Y}_{\mathrm{p}}$ of all genotypes

" $\mathrm{S}$ " characterizes the relative heat tolerance of the genotypes, where $\mathrm{S} \leq 0.5$ is highly heat tolerant, $0.5<\mathrm{S} \leq 1.0$ is moderately heat tolerant, and $\mathrm{S}>1.0$ is heat sensitive.

\section{Results and Discussion}

Twenty-four snap bean and dry bean genotypes were screened over a range of high temperatures and their yield components evaluated, and as expected yield decreased in high-temperature treatments and genotype interaction with treatment was highly significant. Nonuniformity of temperatures and humidity that can occur in growth chamber and some greenhouse environments were minimized in this experiment by spacious greenhouses. However, as is generally the case in common bean, yield among genotypes for the entire study was lower than what is typically seen under field conditions.

Temperatures for the duration of heat treatment were consistent and within $1^{\circ}$ of the set point in T1-T3. Average temperature fell slightly in the two hottest greenhouses (T3 and T4) as outdoor temperatures dropped below freezing. However, in the first $21 \mathrm{~d}$ of heat treatment when reproductive development was most sensitive to high temperatures, temperatures in T3 and T4 were close to the set points with smaller standard deviations. Day temperatures during this period for T3 and T4 averaged $29.94{ }^{\circ} \mathrm{C} \pm 0.73$ and $32.65 \pm 0.84$, respectively, and night temperatures averaged $26.92 \pm 0.74$ and $29.09 \pm 1.45$, respectively. Temperatures had a high degree of uniformity within the greenhouses; thermocouples distributed throughout individual greenhouses at different times gave a mean standard deviation of $\pm 1.29^{\circ} \mathrm{C}$.

The effects of treatment, genotype and the genotype $\times$ treatment interaction were significant $(P \leq 0.0001)$ for the four yield components listed in Table 2 . The only significant differences among replications $(P \leq 0.05)$ occurred between the two replications positioned on the outside wall of the greenhouses when compared to the two replications positioned on the inside wall, suggesting light availability was the factor causing replication to be significant in this experiment. Similarly, plant position within 
Table 1. Market class and source of 24 common bean genotypes evaluated at four temperature regimes in the greenhouses at the Cornell Univ., Geneva, N.Y., in 2002.

\begin{tabular}{|c|c|c|}
\hline Genotype & Market class ${ }^{\mathrm{z}}$ & Source \\
\hline$\overline{\mathrm{A} 55}$ & DB & $\begin{array}{l}\text { International Center for Tropical Agriculture, Cali, } \\
\text { Columbia (CIAT) }\end{array}$ \\
\hline Barrier & SB & Alpha Seeds, Henley on Klip, South Africa \\
\hline Brio & SB & Seminis Vegetable Seeds, Woodland, Calif. \\
\hline Carson & WB & Syngenta Seeds, Golden Valley, Minn. \\
\hline CELRK & DB & $\begin{array}{l}\text { U.S. Dept. of Agriculture, Agricultural Research Service, } \\
\text { Prosser, Wash. }\end{array}$ \\
\hline Contender & $\mathrm{SB} / \mathrm{DB}$ & Heirloom \\
\hline CT70 & SB & Cornell Univ., Geneva, N.Y. \\
\hline G122 & DB & CIAT \\
\hline Haibushi & SB & $\begin{array}{l}\text { Japan International Research Center for Agricultural Sciences, } \\
\text { Tsukuba City, Japan }\end{array}$ \\
\hline HB 1880 & SB & Syngenta Seeds \\
\hline HT 20 & SB & Cornell Univ. \\
\hline HT 24 & SB & Cornell Univ. \\
\hline HT 38 & SB & Cornell Univ. \\
\hline Hystyle & SB & Harris Moran Seed Co., Modesto, Calif. \\
\hline Indeterminate & DB & Univ. of Puerto Rico, Mayaguez \\
\hline \multicolumn{3}{|l|}{ Jamaica Red } \\
\hline Labrador & SB & Seminis Vegetable Seeds \\
\hline Majestic & WB & Syngenta Seeds \\
\hline Opus & SB & Seminis Vegetable Seeds \\
\hline PI 182004 & DB & $\begin{array}{l}\text { U.S. Dept. of Agriculture Western Regional Plant Introduction } \\
\text { Station, Pullman, Wash. (USDA W-6) [origin: Guatemala] }\end{array}$ \\
\hline PI 271998 & DB & USDA W-6 [Spain] \\
\hline PI 307802 & DB & USDA W-6 [El Salvador] \\
\hline PI 309844 & DB & USDA W-6 [Costa Rica] \\
\hline Tío Canela-75 & DB & Zamarano (Panamerican School of Agriculture), Honduras \\
\hline Venture & SB & Syngenta Seeds \\
\hline
\end{tabular}

Table 2. Mean squares for pod number, seed number, mean seed weight and seeds per pod for 24 common bean genotypes evaluated at four temperature regimes in greenhouses at the Cornell Univ., Geneva, N.Y., in 2002.

\begin{tabular}{|c|c|c|c|c|c|}
\hline \multirow[b]{2}{*}{ Source } & \multirow[b]{2}{*}{ df } & \multicolumn{4}{|c|}{ Mean squares } \\
\hline & & $\begin{array}{l}\text { Pod } \\
\text { no. }\end{array}$ & $\begin{array}{c}\text { Seed } \\
\text { no. }\end{array}$ & $\begin{array}{c}\text { Mean } \\
\text { seed wt }\end{array}$ & $\begin{array}{c}\text { Seeds/ } \\
\text { pod }\end{array}$ \\
\hline Genotype (G) & 23 & $109.1^{* * * *}$ & $2605.0^{* * * *}$ & $0.47^{\text {**** }}$ & $29.41^{\text {**** }}$ \\
\hline Treatment $(\mathrm{T})$ & 3 & $2176.0^{* * *}$ & $46866.7^{* * * *}$ & $1.18^{* * * *}$ & $831.7^{* * *}$ \\
\hline Replication (R) & 3 & $14.8^{*}$ & $205.5^{*}$ & $0.32^{*}$ & $7.58^{* * *}$ \\
\hline Plant (P) & 4 & $20.2^{* *}$ & $239.1^{* *}$ & 0.08 & 0.39 \\
\hline $\mathrm{G} \times \mathrm{T}$ & 69 & $34.9^{* * *}$ & $525.0^{* * *}$ & $0.16^{* * *}$ & $6.58^{* * *}$ \\
\hline $\mathrm{G} \times \mathrm{R}$ & 69 & $9.05^{* * *}$ & $111.9^{* * *}$ & $0.14^{* *}$ & $1.61^{* *}$ \\
\hline $\mathrm{G} \times \mathrm{P}$ & 90 & 4.80 & 56.63 & 0.07 & 0.93 \\
\hline $\mathrm{T} \times \mathrm{R}$ & 9 & $32.29^{* * *}$ & $453.6^{* * * *}$ & 0.08 & $4.52^{* * *}$ \\
\hline Error & 1604 & 3.82 & 46.66 & 0.08 & 0.94 \\
\hline
\end{tabular}

*,**,*** Significance at $P \leq 0.01, P \leq 0.001$, or $P \leq 0.0001$, respectively.

each group of five plants was significant $(P \leq 0.05)$ for pod number and seed number due to differences between the plants positioned close to the light gap at the interior of the bench and those plants receiving less light on the edge benches.

As expected, values for yield components among heat susceptible controls 'A55' (Shonnard and Gepts, 1994), 'Majestic' (Monterroso and Wien, 1988), and 'Labrador' (M.H. Dickson, personal communication) in high-temperature treatments ranked low among all genotypes. Many genotypes previously reported as heat tolerant displayed tolerant reactions in this experiment, including 'G122', 'Venture', and to some extent PI 271998. The exceptions were three genotypes previously reported as heat tolerant, 'Haibushi', 'IJR', and 'Tío Canela-75', which were susceptible to high temperatures in this experiment. Disparities between greenhouse and field conditions may have contributed to these unexpected results. Additionally, this experiment emphasized high night temperatures, whereas these genotypes may be more tolerant to conditions of high day temperatures only. The performances of 'Haibushi', 'IJR', and 'Tío Canela-75' in this study suggest tolerance to high day temperature may be a separate trait in common bean from tolerance to high night temperature.

Seed number in all genotypes except 'Hystyle' was significantly lower in T4 (Table 3 ) as compared to other treatments. High temperatures reduced seed number $16 \%$ in T3 and $83 \%$ in T4 over all genotypes. Comparing the mean of $\mathrm{T} 3$ and $\mathrm{T} 4$ with the mean of $\mathrm{T} 1$ and $\mathrm{T} 2$, high temperatures decreased seed number $70 \%$ among heat-sensitive genotypes ('A55', 'Labrador', 'Majestic', 'IJR') as compared to $29 \%$ in heat-tolerant genotypes. Pod number was significantly lower over all genotypes only in T4 $63 \%$ reduction), decreasing $97 \%$ in heat-sensitive genotypes and $34 \%$ in heat-tolerant genotypes ('Brio', 'Carson', 'G122', 'HB1880', 'HT 38', 'Venture'). Similarly, mean seed weight was significantly lower in T4 only, decreasing $47 \%$ over all genotypes, $88 \%$ in heatsensitive genotypes and $25 \%$ in heat-tolerant genotypes. Finally, seeds per pod was significantly different for all four treatments, increasing $6 \%$ in T2 and decreasing $20 \%$ in T3, $73 \%$ in T4, 66\% in heat-sensitive genotypes (mean of $\mathrm{T} 1+\mathrm{T} 2$ vs. mean of $\mathrm{T} 3+$ $\mathrm{T} 4$ ), and $31 \%$ in heat-tolerant genotypes. 


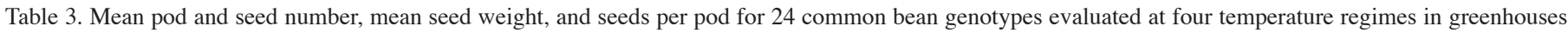
at the Cornell Univ., Geneva, N.Y., in 2002. Also included are percent reduction of lowest value from highest value among treatments and heat susceptibility index values.

\begin{tabular}{|c|c|c|c|c|c|c|c|c|c|c|c|c|c|c|c|c|c|c|c|c|c|c|c|}
\hline \multirow[b]{2}{*}{ Genotype } & \multicolumn{6}{|c|}{ Pod number } & \multicolumn{6}{|c|}{ Seed number } & \multicolumn{6}{|c|}{ Mean seed weight } & \multicolumn{5}{|c|}{ Seed/ pod } \\
\hline & $\mathbf{T 1}^{\mathbf{2}}$ & $\mathbf{T} 2$ & T3 & T4 & $\mathbf{P R}^{y}$ & $\mathbf{S}^{\mathbf{x}}$ & T1 & T2 & T3 & T4 & PR & $\bar{S}$ & T1 & T2 & T3 & T4 & PR & $\bar{S}$ & T1 & $\mathbf{T 2}$ & T3 & T4 PR & S \\
\hline $\mathbf{A 5 5}$ & $4.5 b^{w}$ & $5.0 \mathrm{~b}$ & $6.7 \mathrm{a}$ & $0.1 \mathrm{c}$ & 99 & 0.94 & 21.5 ab & 22.7 a & $19.1 \mathrm{~b}$ & $0.1 \mathrm{c}$ & 99 & 1.13 & $0.13 \mathrm{a}$ & 0.13 a & $0.11 \mathrm{~b}$ & 0.01 c & 92 & 1.90 & $4.95 \mathrm{a}$ & $4.7 \mathrm{a}$ & $2.93 \mathrm{~b}$ & 0.10 c 98 & 1.47 \\
\hline Barrier & $7.9 \mathrm{a}$ & $6.8 \mathrm{a}$ & $7.9 \mathrm{a}$ & $0.6 \mathrm{c}$ & 92 & 1.38 & 29.3 a & $25.9 \mathrm{a}$ & $25.8 \mathrm{a}$ & $0.9 \mathrm{~b}$ & 97 & 1.03 & $0.14 \mathrm{at}$ & $0.11 \mathrm{ab}$ & $0.13 \mathrm{a}$ & 0.05 b & 64 & 0.99 & $2.91 \mathrm{c}$ & $4.31 \mathrm{a}$ & $3.43 \mathrm{~b}$ & $0.65 d 85$ & 0.93 \\
\hline Brio & $4.3 \mathrm{a}$ & $6.7 \mathrm{~b}$ & $8.5 \mathrm{a}$ & $2.3 \mathrm{~d}$ & 99 & 0.05 & $17.1 \mathrm{~b}$ & $29.8 \mathrm{a}$ & 28.4 a & $4.8 \mathrm{c}$ & 84 & 0.59 & 0.08 b & $.09 \mathrm{~b}$ & $0.11 \mathrm{a}$ & 0.07 b & 22 & -0.21 & $3.01 \mathrm{~b}$ & $4.39 \mathrm{a}$ & $3.37 \mathrm{~b}$ & $1.21 \mathrm{c} 72$ & 0.82 \\
\hline Carson & $7.3 \mathrm{~b}$ & $8.1 \mathrm{ab}$ & $9.0 \mathrm{a}$ & $5.6 \mathrm{~b}$ & 38 & 0.16 & $25.3 \mathrm{~b}$ & 30.7 a & $32.5 \mathrm{a}$ & $9.6 \mathrm{c}$ & 70 & 0.49 & $0.16 \mathrm{a}$ & 0.17 a & $0.15 \mathrm{a}$ & $0.11 \mathrm{~b}$ & 35 & 0.75 & $3.47 \mathrm{a}$ & $3.80 \mathrm{a}$ & $3.53 \mathrm{a}$ & 1.59 b 58 & 0.63 \\
\hline CELRK & $5.2 \mathrm{~b}$ & $5.1 \mathrm{ab}$ & $6.6 \mathrm{~b}$ & $6.0 \mathrm{ab}$ & 21 & -0.53 & $14.1 \mathrm{~b}$ & $16.1 \mathrm{a}$ & $17.1 \mathrm{a}$ & $9.5 \mathrm{c}$ & 45 & 0.24 & $0.41 \mathrm{a}$ & 0.38 a & $0.29 \mathrm{~b}$ & $0.22 \mathrm{c}$ & 46 & 1.25 & $2.82 \mathrm{a}$ & $2.99 \mathrm{a}$ & $2.66 \mathrm{a}$ & 1.75 b 41 & 0.52 \\
\hline Contender & $5.4 \mathrm{a}$ & $5.3 \mathrm{a}$ & $4.7 \mathrm{a}$ & $2.7 \mathrm{a}$ & 50 & 1.02 & $17.8 \mathrm{ab}$ & $19.5 \mathrm{a}$ & $14.7 \mathrm{~b}$ & $4.2 \mathrm{c}$ & 78 & 0.99 & $0.35 \mathrm{a}$ & 35 a & $0.28 \mathrm{ab}$ & O 27 b & 23 & 0.75 & $3.24 \mathrm{ab}$ & $3.74 \mathrm{a}$ & $2.86 \mathrm{~b}$ & 1.20 c 68 & 0.90 \\
\hline CT 70 & $7.8 \mathrm{a}$ & $7.4 \mathrm{ab}$ & $6.6 \mathrm{~b}$ & $3.1 \mathrm{c}$ & 60 & 1.18 & $34.0 \mathrm{a}$ & 29.9 & $22.0 \mathrm{ab}$ & $6.1 \mathrm{~d}$ & 82 & 1.12 & 0.17 a & 0.16 a & 0.16 a & 0.09 b & 47 & 0.86 & $4.40 \mathrm{a}$ & $4.06 \mathrm{a}$ & $3.29 \mathrm{a}$ & 1.78 c 60 & 0.86 \\
\hline G122 & $6.1 \mathrm{~b}$ & $6.6 \mathrm{ab}$ & $7.6 \mathrm{a}$ & $7.2 \mathrm{a}$ & 19 & -0.53 & $19.8 \mathrm{~b}$ & $22.2 \mathrm{ab}$ & $24.8 \mathrm{a}$ & $12.6 \mathrm{c}$ & 49 & 0.22 & $0.21 \mathrm{ab}$ & $0.23 \mathrm{a}$ & $0.20 \mathrm{~b}$ & $0.16 \mathrm{c}$ & 30 & 0.64 & $3.31 \mathrm{a}$ & $3.44 \mathrm{a}$ & $3.31 \mathrm{a}$ & $1.69 \mathrm{~b} 51$ & 0.56 \\
\hline Haibushi & $8.8 \mathrm{a}$ & $6.7 \mathrm{~b}$ & $6.3 \mathrm{~b}$ & $.3 \mathrm{c}$ & 96 & 1.86 & $40.5 a$ & $28.8 \mathrm{~b}$ & $21.0 \mathrm{~b}$ & $0.8 \mathrm{c}$ & 98 & 1.37 & 0.14 a & 0.12 a & $1.17 \mathrm{a}$ & $0.02 \mathrm{a}$ & 86 & 2.99 & $4.72 \mathrm{a}$ & $4.45 \mathrm{a}$ & $3.31 \mathrm{~b}$ & 0.63 c 87 & 1.22 \\
\hline HB1880 & $7.6 \mathrm{a}$ & $6.6 \mathrm{~b}$ & $6.4 \mathrm{~b}$ & $3.8 \mathrm{c}$ & 50 & 0.91 & $36.4 \mathrm{a}$ & $30.1 \mathrm{~b}$ & $26.1 \mathrm{~b}$ & $7.3 \mathrm{c}$ & 80 & 0.99 & 0.12 a & $0.12 \mathrm{a}$ & $0.12 \mathrm{a}$ & 0.13 a & 0 & -0.15 & 4.87 & $56 \mathrm{ab}$ & $4.13 \mathrm{~b}$ & 1.78 c 63 & 0.80 \\
\hline HT 20 & $7.1 \mathrm{a}$ & $7.4 \mathrm{a}$ & $6.6 \mathrm{a}$ & $5.25 \mathrm{~b}$ & 29 & 0.60 & $28.2 \mathrm{a}$ & 28.4 a & $22.1 \mathrm{~b}$ & $9.4 \mathrm{c}$ & 67 & 0.89 & 0.19 ab & $20 \mathrm{a}$ & 0.18 ab & 0.17 b & 15 & 0.36 & $4.02 \mathrm{a}$ & $3.86 \mathrm{a}$ & $3.40 \mathrm{~b}$ & 1.77 c 56 & 0.74 \\
\hline HT 24 & $9.5 \mathrm{a}$ & $9.2 \mathrm{a}$ & $9.4 \mathrm{a}$ & $3.1 \mathrm{~b}$ & 67 & 1.08 & 26.9 & $25.1 \mathrm{ab}$ & $21.4 \mathrm{~b}$ & $4.7 \mathrm{c}$ & 83 & 1.00 & 0.21 a & $0.20 \mathrm{a}$ & $0.20 \mathrm{a}$ & $0.11 \mathrm{~b}$ & 48 & 0.86 & $2.92 \mathrm{a}$ & $2.90 \mathrm{a}$ & $2.36 \mathrm{~b}$ & 0.88 c 70 & 0.95 \\
\hline HT 38 & $6.8 \mathrm{~b}$ & $7.1 \mathrm{ab}$ & $8.4 \mathrm{a}$ & $5.5 \mathrm{c}$ & 35 & 0.01 & $22.4 \mathrm{~b}$ & 28.5 a & $26.7 \mathrm{a}$ & $10.3 \mathrm{c}$ & 64 & 0.54 & 0 a & $\mathbf{a}$ & a & 0.12 b & 25 & 0.46 & $3.41 \mathrm{~b}$ & $3.96 \mathrm{a}$ & 3.32 b & 1.84 c 54 & 0.64 \\
\hline Hystyle & $4.8 \mathrm{a}$ & $4.3 \mathrm{a}$ & $4.5 \mathrm{a}$ & $1.0 \mathrm{~b}$ & 80 & 1.30 & $9.5 \mathrm{a}$ & $10.2 \mathrm{a}$ & $12.1 \mathrm{a}$ & $1.3 \mathrm{~b}$ & 89 & 0.64 & $0.04 b$ & 0.08ab & $0.09 \mathrm{a}$ & $0.05 \mathrm{~b}$ & 44 & -0.59 & $2.00 \mathrm{ab}$ & $1.62 \mathrm{~b}$ & $2.46 \mathrm{a}$ & 0.46 c 81 & 0.41 \\
\hline IJR & $5.7 \mathrm{a}$ & $4.4 \mathrm{~b}$ & $3.1 \mathrm{c}$ & $0.2 \mathrm{~d}$ & 96 & 2.19 & $15.2 \mathrm{a}$ & $11.8 \mathrm{~b}$ & $5.1 \mathrm{c}$ & $0.4 \mathrm{~d}$ & 98 & 1.59 & $0.21 \mathrm{a}$ & 0.19 a & 0.14 b & $0.03 \mathrm{c}$ & 86 & 2.03 & $2.74 \mathrm{a}$ & $\mathbf{a}$ & $1.34 \mathrm{~b}$ & 0.18 c 96 & 1.56 \\
\hline Labrador & $7.0 \mathrm{~b}$ & $8.1 \mathrm{a}$ & $5.9 \mathrm{c}$ & $0.5 \mathrm{~d}$ & 94 & 1.90 & $25.1 \mathrm{~b}$ & 31.7 a & $12.2 \mathrm{c}$ & $0.7 \mathrm{~d}$ & 98 & 1.55 & $\mathbf{0}$ & 0 & 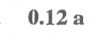 & 0. & 75 & 1.12 & $3.47 \mathrm{a}$ & 3.87 a & $2.23 \mathrm{~b}$ & 0.45 c 88 & 1.36 \\
\hline Majestic & $6.4 \mathrm{a}$ & $6.9 \mathrm{a}$ & $4.7 \mathrm{~b}$ & $0.0 \mathrm{c}$ & 100 & 2.13 & 16.2 & 19 & $11.4 \mathrm{~b}$ & $0.0 \mathrm{c}$ & 100 & 1.36 & $0.19 a$ & 0.18 a & $0.14 b$ & $0.0 \mathrm{c}$ & 100 & 2.20 & $2.53 \mathrm{a}$ & $2.75 \mathrm{a}$ & $2.32 \mathrm{a}$ & 0.0 b 100 & 1.20 \\
\hline Opus & $7.2 \mathrm{a}$ & $7.0 \mathrm{a}$ & $8.2 \mathrm{a}$ & $2.4 \mathrm{~b}$ & 71 & 0.82 & $21.0 \mathrm{~b}$ & 31.4 a & 27.7 a & $3.45 \mathrm{c}$ & 89 & 0.81 & $0.12 \mathrm{a}$ & $0.13 \mathrm{a}$ & $0.14 \mathrm{a}$ & $0.08 \mathrm{a}$ & 43 & -0.27 & $3.0 \mathrm{~b}$ & 5.19 & $54 \mathrm{ab}$ & 0.97 c 81 & 0.96 \\
\hline PI 182004 & $5.8 \mathrm{a}$ & $6.8 \mathrm{a}$ & $6.1 \mathrm{a}$ & $1.4 \mathrm{~b}$ & 80 & 1.34 & 17. & $20.5 \mathrm{a}$ & 10.00 & $1.9 \mathrm{c}$ & 91 & 1.02 & $0.29 \mathrm{a}$ & $0.28 \mathrm{a}$ & $0.27 \mathrm{a}$ & $0.12 \mathrm{~b}$ & 59 & 1.11 & $2.97 \mathrm{a}$ & $3.06 \mathrm{a}$ & $2.78 \mathrm{a}$ & 0.76 b 75 & 0.89 \\
\hline PI 271998 & $5.2 \mathrm{~b}$ & $5.5 \mathrm{~b}$ & $6.6 \mathrm{a}$ & $1.1 \mathrm{c}$ & 83 & 0.90 & $17.6 \mathrm{a}$ & $17.0 \mathrm{a}$ & $17.0 \mathrm{a}$ & $1.4 \mathrm{~b}$ & 92 & 0.93 & $0.39 \mathrm{a}$ & $0.31 \mathrm{~b}$ & $0.30 \mathrm{~b}$ & $0.17 \mathrm{c}$ & 56 & 1.16 & $3.45 \mathrm{a}$ & $3.20 \mathrm{a}$ & $2.56 \mathrm{~b}$ & 0.66 c 81 & 1.11 \\
\hline PI 307802 & $7.2 \mathrm{a}$ & $8.4 \mathrm{a}$ & $7.3 \mathrm{a}$ & $1.3 \mathrm{~b}$ & 85 & 1.47 & $31.7 \mathrm{~b}$ & $37.8 \mathrm{a}$ & $19.9 \mathrm{c}$ & $3.2 \mathrm{~d}$ & 92 & 1.34 & $0.16 \mathrm{a}$ & $0.16 \mathrm{a}$ & $0.13 \mathrm{~b}$ & $0.04 \mathrm{c}$ & 75 & 1.66 & $4.47 \mathrm{a}$ & $4.50 \mathrm{a}$ & $2.30 \mathrm{~b}$ & 1.12 c 75 & 1.33 \\
\hline PI 309844 & $7.9 \mathrm{a}$ & $8.8 \mathrm{a}$ & $9.1 \mathrm{a}$ & $2.4 \mathrm{~b}$ & 74 & 1.01 & $44.3 \mathrm{a}$ & $46.2 \mathrm{a}$ & 38.2 b & $3.4 \mathrm{c}$ & 93 & 1.08 & 0.14 a & 0.14 a & 0.15 a & $0.08 \mathrm{~b}$ & 47 & 0.63 & $5.58 \mathrm{a}$ & $5.33 \mathrm{a}$ & $4.29 \mathrm{~b}$ & 0.71 c 87 & 1.16 \\
\hline Tío Canela & $9.1 \mathrm{a}$ & $8.3 \mathrm{a}$ & $8.2 \mathrm{a}$ & $0.0 \mathrm{~b}$ & 100 & 1.73 & $37.6 \mathrm{a}$ & 30.8 b & $21.4 \mathrm{c}$ & $0.0 \mathrm{~d}$ & 100 & 1.37 & 0.13 b & 0.13 b & 0.16 a & $0.0 \mathrm{c}$ & 100 & 1.36 & $4.15 \mathrm{a}$ & $3.78 \mathrm{a}$ & $2.66 \mathrm{~b}$ & 0.0 c 100 & 1.43 \\
\hline Venture & $6.1 \mathrm{~b}$ & $6.1 \mathrm{~b}$ & $7.3 \mathrm{a}$ & $3.3 \mathrm{c}$ & 46 & 0.42 & $24.8 \mathrm{a}$ & 24.7 a & $21.9 \mathrm{a}$ & $4.9 \mathrm{~b}$ & 80 & 0.92 & $0.20 \mathrm{ab}$ & $0.22 \mathrm{a}$ & $0.20 \mathrm{ab}$ & $0.18 \mathrm{~b}$ & 18 & 0.34 & $4.11 \mathrm{a}$ & $4.15 \mathrm{a}$ & $3.07 \mathrm{~b}$ & 1.34 c 68 & 1.00 \\
\hline MEAN & $6.62 \mathrm{a}$ & $6.79 \mathrm{a}$ & $6.89 \mathrm{a}$ & $2.49 \mathrm{~b}$ & 97 & & 24.37 b & 25.87 a 2 & $21.04 \mathrm{c}$ & $4.26 \mathrm{~d}$ & 84 & & $\mathbf{a}$ & (1) & $0 \mathbf{a}$ & 0.10 b & 52 & & $59 \mathrm{~b}$ & $3.80 \mathrm{a}$ & $.97 \mathrm{c}$ & 0.99 d 75 & \\
\hline
\end{tabular}

zT $1=24^{\circ} \mathrm{C}$ day $/ 21{ }^{\circ} \mathrm{C}$ night; $\mathrm{T} 2=27^{\circ} \mathrm{C} / 24^{\circ} \mathrm{C} ; \mathrm{T} 3=30^{\circ} \mathrm{C} / 27^{\circ} \mathrm{C} ; \mathrm{T} 4=33^{\circ} \mathrm{C} / 30^{\circ} \mathrm{C}$

yPR= percent reduction

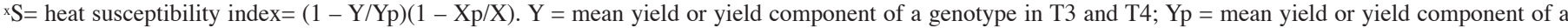
genotype in $\mathrm{T} 1$ and $\mathrm{T} 2 ; \mathrm{X}=$ mean $\mathrm{Y}$ of all genotypes; $\mathrm{Xp}=$ mean $\mathrm{Yp}$ of all genotypes.

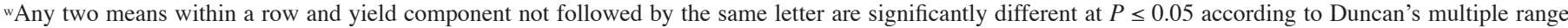
test.

Pod number decreased in heat-sensitive genotypes in all three high-temperature treatments primarily as a result of abscission of buds and flowers (data not shown), a phenomenon reported by others (Gross and Kigel, 1994; Ofir et al., 1993; Porch, 2001). However, pod number increased through T3 in 12 genotypes, many of which were heat tolerant, such as 'Carson' and 'HT 38 ', and may be the result of an increase in the number of reproductive organs produced, as seen by Konsens et al. (1991) in common bean and by Prasad et al. (1999) in groundnut (Arachis hypogaea L.).

The heat susceptibility index (S) was calculated as a relative measure of yield stability under heat stress that removes variation in genotype yield potential from consideration (Fischer and Maurer, 1978). 'Hystyle' was an example of a genotype with low yield potential but stable yields under heat stress; this genotype exhibited stable seed and pod production but yield component values were consistently low compared to other genotypes.

No genotypes had $\mathrm{S} \leq 0.50$ for all yield components considered. Genotypes with values of $\mathrm{S} \leq 1.0$ for all yield components were 'Brio', 'Carson', 'G122', 'HB 1880', 'HT 20', 'HT 38', 'Opus', and 'Venture'. Genotypes with $\mathrm{S} \leq 0.50$ for seed number were 'Carson' ( $\mathrm{S}=0.49)$, 'CELRK' (0.24), and 'G122' (0.22); for pod number were 'Brio' (0.05), 'Carson' (0.16), 'CELRK' $(-0.53)$, 'G122' (-0.53), 'HT 38' (0.01), and 'Venture' (0.42); for mean seed weight were 'Brio' (-0.21), 'HB 1880' (-0.15), 'HT 20' (0.36), 'HT 38' (0.46), 'Hystyle' $(-0.59)$, 'Opus' (-0.27), and 'Venture' (0.34). Finally, for seeds per pod, 'Hystyle' (0.41) was the only genotype with $\mathrm{S} \leq 0.50$. Heat-sensitive genotypes according to the heat susceptibility index included 'Haibushi', 'IJR', 'Labrador', 'Majestic', and 'Tío Canela-75'.

Future studies and germplasm screens attempting to identify material for improvement of heat tolerance in common bean should use a temperature regime similar to that in $\mathrm{T} 3\left(30^{\circ} \mathrm{C}\right.$ day/ $27^{\circ} \mathrm{C}$ night). In that treatment, moderately heat-tolerant genotypes and genotypes possessing yield stability, such as 'Barrier' and 'Hystyle', were identified; higher temperatures would not resolve the relative heat tolerance or sensitivity of most common bean materials. However, if known heat-tolerant material is being studied, a temperature regime close to that of $\mathrm{T} 4$ would be most informative.

Differential heat-tolerance reactions were observed that may allow for the combination of nonallelic heat-tolerance genes. In Fig. 1a, displaying regression lines across the four temperature treatments, 'HB 1880' and 'Venture' have large negative slopes for seed number, in contrast to 'Carson' and 'G122', which have relatively small slopes for this yield component. Low-yielding genotypes able to maintain mean seed weight under high temperatures may be candidates for improvement of pod retention 


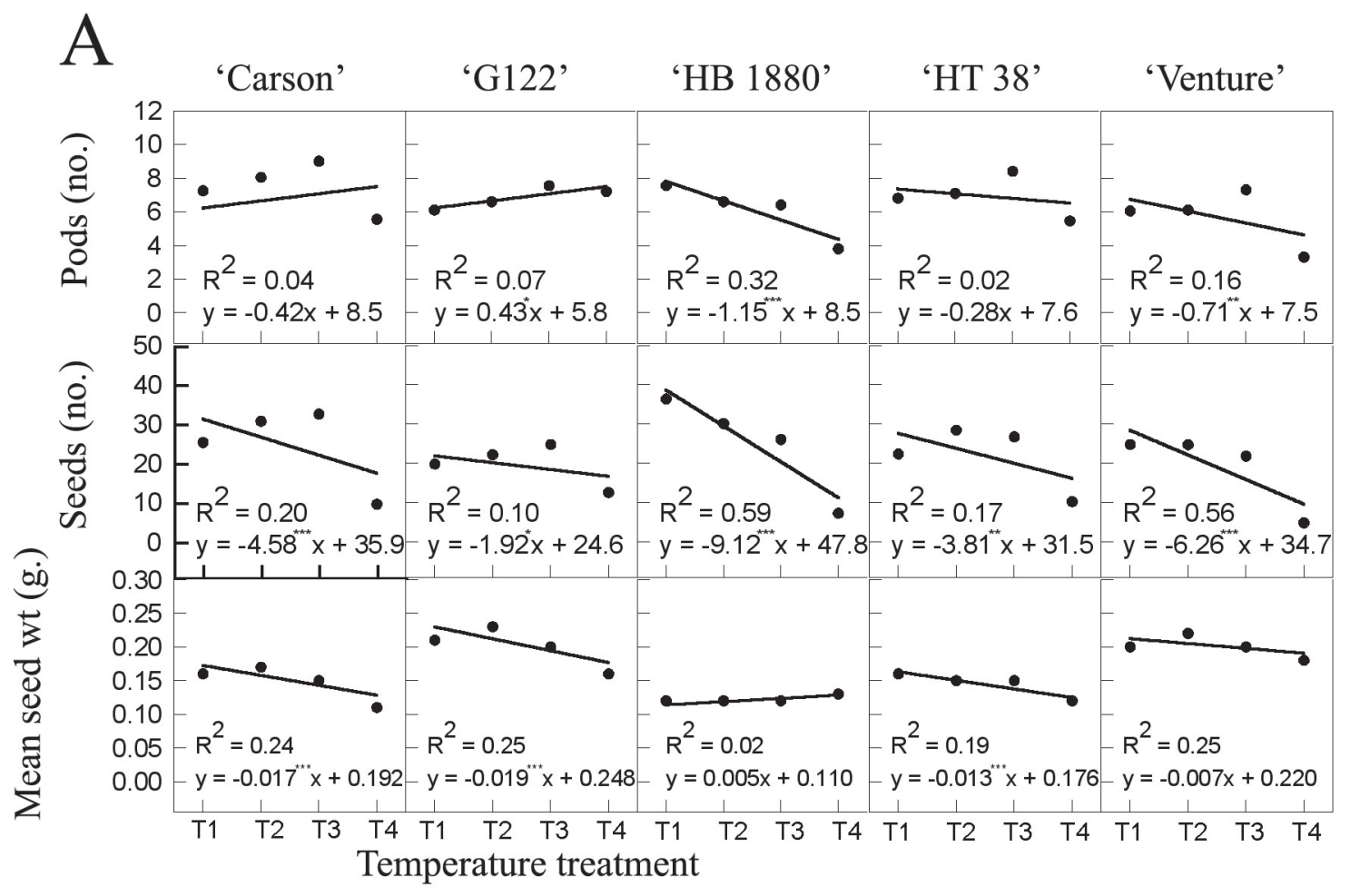

B

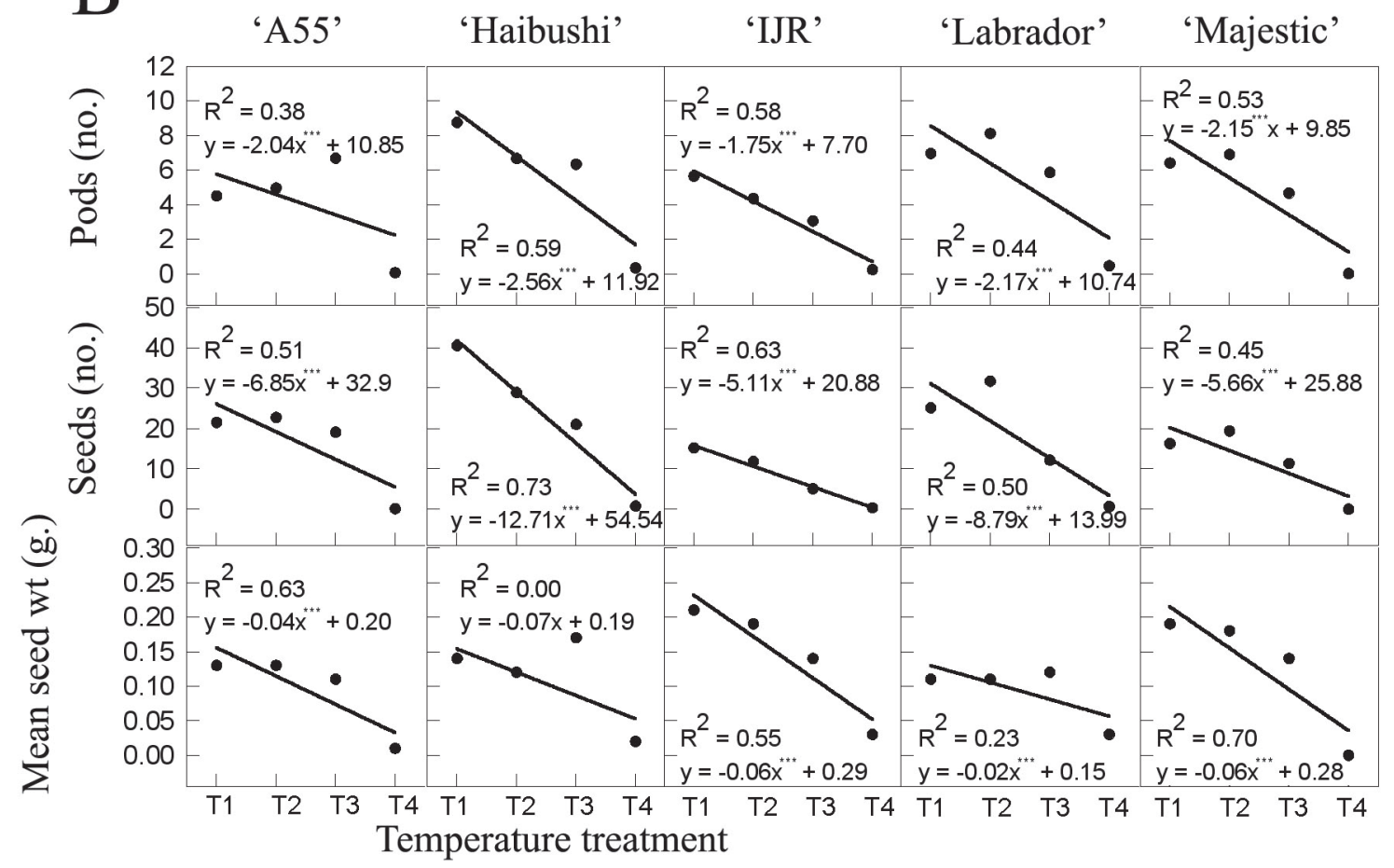

Fig. 1. Regression lines and treatment means for select genotypes and yield components. (A) Heat-tolerant genotypes; (B) heat-sensitive genotypes. Genotypes were evaluated under four temperature treatments in greenhouses at the Cornell Univ., Geneva, in $2002.1: \mathrm{T} 1=24^{\circ} \mathrm{C}$ day $/ 21^{\circ} \mathrm{C}$ night; 2 : $\mathrm{T} 2=27^{\circ} \mathrm{C} / 24^{\circ} \mathrm{C} ; 3$ : $\mathrm{T} 3=30{ }^{\circ} \mathrm{C} / 27^{\circ} \mathrm{C} ; 4: \mathrm{T} 4=33{ }^{\circ} \mathrm{C} / 30^{\circ} \mathrm{C}$. 
and seed set; these included 'Haibushi' and 'Labrador', shown in Fig. 1b. 'Majestic' and 'IJR', however, have very negative slopes for all yield components, which may indicate greater somatic heat susceptibility as mean seed weight decreased even though fewer seeds were set. Studies in cotton (Gossypium hirsutum L.) (Garay and Barrow, 1988) and cowpea (Vigna unguiculata) (Morfo and Hall, 1992) have improved heat-sensitive genotypes using genotypes with heat-tolerant pollen traits. Presuming pollen germination, pollen tube growth, and/or fertilization were relatively more successful in genotypes with stable pod and seed sets, as seen in 'Carson', and 'HT 38' in Fig. 1a, these traits could be utilized for improvement of varieties with reduced pod and seed set or in combination with other heat-tolerance traits.

\section{Literature Cited}

Baiges, S., J.S. Beaver, P.N. Miklas, and J.C. Rosas. 1996. Evaluation and selection of dry beans for heat tolerance. Annu. Rpt. Bean Improvement Coop. 39:95-96.

Boodley, J.W. and R. Sheldrake, Jr. 1972. Cornell peat-lite mixes for commercial plant growing. Cornell Info. Bul. 43:1-8.

Dickson, M.H., and M.A. Boettger. 1984. Effect of high and low temperature on pollen germination and see set in snap beans Phaseolus vulgaris L. J. Amer. Soc. Hort. Sci. 109:372-374.

Ehlers, J.D. and A.E. Hall. 1996. Genotypic classification of cowpea based on response to heat and photoperiod. Crop Sci. 36:673-679.

Fischer, R.A. and R. Maurer. 1978. Drought resistance in spring wheat cultivars. I. Grain yield response. Austral. J. Agr. Res. 29:897-912.

Garay, B.R. and J.R. Barrow. 1988. Pollen selection for heat tolerance in cotton. Crop Sci. 28: 857-859.

Gross, Y. and J. Kigel. 1994. Differential sensitivity to high temperature of stages in the reproductive development of common bean (Phaseolus vulgaris L.). Field Crops Res. 36:201-212.

Halterlein, A.J., C.D. Clayberg, and I.D. Teare. 1980. Influence of high temperature on pollen grain viability and pollen tube growth in the styles of Phaseolus vulgaris L. J. Amer. Soc. Hort. Sci. 105:12-14.

Ismail, A.M. and A.E. Hall. 1998. Positive and negative effects of heattolerance genes studied using closely related cowpea lines. Crop Sci. 38:381-390

Konsens, I., M. Ofir, and J. Kigel. 1991. The effect of temperature on the production and abscission of flowers and pods in snap bean (Phaseolus vulgaris L.). Ann. Bot. 67:391-399.

Monterroso, V.A. and H.C. Wien. 1988. Effect of high temperature on pod set of Phaseolus vulgaris. Annu. Rpt. Bean Improvement Coop. $31: 160$.

Monterroso, V.A. and H.C. Wien. 1990. Flower and pod abscission due to heat stress in beans. J. Amer. Soc. Hort. Sci. 115:631-634.

Morfo, K.O. and A.E. Hall. 1992. Inheritance of heat tolerance during pod set in cowpea. Crop Sci. 32:912-918.

Myers, J.R. and J.R. Baggett. 1999. Improvement of snap beans, p. 289-329. In: S.P. Singh (ed.). Common bean improvement in the twenty-first century. Kluwer Academic Publishers, Dordrecht, The Netherlands.

Omrod, D.P., C.J. Woolley, G.W. Eaton, and E.H. Stobbe. 1967. Effect of temperature on embryo sac development in Phaseolus vulagris L. Can. J. Bot. 45:948-950.

Ofir, M., Y. Gross, F. Bangerth, and J. Kigel. 1993. High temperature effects on pod and seed production as related to hormone levels and abscission of reproductive structures in common bean (Phaseolus vulgaris L.). Scientia Hort. 55:201-211.

Prasad, P.V., P.Q. Craufurd, and R.J. Summerfield. 1999. Sensitivity of peanut to timing of heat stress during reproductive development. Crop Sci. 39:1352-1357.

Porch, T.G. 2001. Genetics and applications of heat tolerance in common bean. PhD Diss. Cornell Univ., Ithaca, N.Y.

Porch, T.G. and M. Jahn. 2001. Effects of high-temperature stress on microsporogenesis in heat-sensitive and heat-tolerant genotypes of Phaseolus vulgaris. Plant, Cell Environ. 24:723-731.

Rosas J.C., O.I. Varela, and J.S. Beaver. 1997. Registration of 'Tío Canela-75-75' small red bean (race Mesoamerica). Crop Sci. 34:3791.

SAS Institute. 1997. SAS user's guide. SAS Inst., Cary, N.C.

Shonnard, G.C. and P. Gepts. 1994. Genetics of heat tolerance during reproductive development in common bean. Crop Sci. 34:1168-1175.

Suzuki, K., T. Tsukaguchi, H. Takeda, and Y. Egawa. 2001. Decrease of pollen stainability of green bean at high temperatures and relationship to heat tolerance. J. Amer. Soc. Hort. Sci. 126:571-574.

Weaver, M.L., D.W. Burke, M.J. Slibernagel, and H. Timm. 1985. Pollen staining and high-temperature tolerance of bean. J. Amer. Soc. Hort. Sci. 110:797-799.

Wien, H.C. and H.M. Munger. 1972. Heat-tolerant Phaseolus vulgaris. Annu. Rpt. Bean Improvement Coop. 15:97-98. 